\title{
Influencias altiplánicas en San Pedro de Atacama
}

Mario Orellana ${ }^{1}$

\section{Consideraciones generales: Objetivos y metodología}

El presente informe adelanta parte de los resultados de una investigación aprobada por el Departamento de Desarrollo Científico de la Universidad de Chile. El análisis que hacemos de las influencias altiplánicas en la región de San Pedro de Atacama y, en especial, de la civilización Tiwanaku, no tiene como objetivo central individualizar artefactos y rasgos culturales altiplánicos, encontrados en el hábitat de los antiguos atacameños, como ejemplo de contactos aislados, o como producto de un proceso unidireccional de difusión o de colonización proveniente de las tierras altas. Lo que pretendemos es describir e interpretar el proceso dinámico intercultural que se produjo entre Tiwanaku y el Complejo Cultural San Pedro (o atacameño).

Ahora bien, el estudio de los fenómenos de interacción entre diferentes sociedades tiene que apoyarse en datos empíricos. Por esta razón, como objetivo más particular, nos interesa saber cuándo llegaron los artefactos y rasgos atribuidos a Tiwanaku (incluyendo las ideas y las creencias), es decir, queremos pensar la temática cronológica del contacto cultural Altiplano-San Pedro de Atacama, en qué siglo se inició y en qué otros adquirió mayor fluidez el proceso de relaciones interculturales. El problema cronológico está en íntima relación con el problema contextual o de asociaciones. Los rasgos altiplánicos de cualquier tipo y complejidad se incorporaron a una cultura; fueron usados por grupos sociales de diferentes condiciones económicas e intelectuales; se distribuyeron, manteniéndose en algunos casos iguales o transformándose (procesos de asimilación y de transculturación). El análisis científico de los contextos culturales, provenientes de las tumbas excavadas en San Pedro de Atacama y sus alrededores,

1 Centro de Estudios Humanísticos, Facultad de Ciencias Físicas y Matemáticas, Universidad de Chile. Ejército 333, Santiago, CHILE. nos permitirá saber con exactitud a qué ambientes socioculturales llegaron los artefactos y las ideas extranjeras, en qué cantidad están presentes, en qué tipos de cementerios y en qué ayllu de San Pedro y sus alrededores se adaptaron. También pretendemos saber quiénes (qué etnias) trajeron estos artefactos: ¿Fueron los propios habitantes de la región de San Pedro de Atacama los que se trasladaron a diferentes lugares del altiplano y, al volver con productos altiplánicos, iniciaron el proceso de intercontactos, o fueron grupos altiplánicos los que bajaron el fértil valle de San Pedro de Atacama trayendo técnicas, ideas y artefactos de diferente tipo? ¿Son aplicables en esta clase de investigación las teorías, enunciadas por otros investigadores, de "verticalidad" (Murra 1972), de "red de intercambio" (Browman 1980), de "esfera de interacción" (Berenguer et al. 1980), etc.? Este último aspecto de la investigación está relacionado con los trabajos de los antropólogos físicos y con la importancia que ellos le dan al rasgo cultural de la deformación craneana (Munizaga 1969).

Teniendo en cuenta lo anterior, en la presente comunicación daremos a conocer algunas conclusiones provisorias (por lo tanto, hipótesis) que nos parecen significativas.

a) Un conjunto importante de restos culturales que contienen rasgos Tiwanaku y que, en algunos casos, son piezas importadas, se sitúan cronológicamente en la segunda mitad del primer milenio de la era cristiana, e incluso, algunos de ellos se fechan hacia 1000 DC.

Esta nueva posición cronológica es interesante de analizar, puesto que en la década de 1970 algunos arqueólogos habían defendido una cronología más temprana, haciendo reroceder la llegada de restos y rasgos Tiwanaku antes de 500 DC (Ponce 1972; Bittmann et al. 1978).

Además, un conjunto de restos del más puro estilo Tiwanaku (huesos pirograbados, algunos tiestos alfareros, tejidos, vasos de madera prosopomorfos 
y algunas tabletas para rape) servía incluso de argumento para organizar una hipótesis muy novedosa: ciertos rasgos que se reconocían como perteneciendo al estilo clásico Tiwanaku habían tenido su origen en San Pedro de Atacama (Le Paige 1965; Serracino 1980).

b) La nueva hipótesis cronológica que proponemos replantea la problemática de Tiwanaku y las influencias altiplánicas en la región de San Pedro de Atacama, e influye en el reanálisis de la Fase II y de los comienzos de la Fase III de la cultura agroalfarera-pastoril de San Pedro.

En los últimos años, apoyados en algunas fechas $\mathrm{C}^{14}$, algunos investigadores habían postulado el desarrollo inicial de la Fase II hacia los comienzos de la era cristiana, coincidiendo con la llegada de restos de Tiwanaku alrededor de 300 DC (Bittmann et al. 1978). Preparado por una etapa temprana (caracterizada por los restos de Toconao Oriente y de otros ayllu de San Pedro de Atacama) entre 500 AC y 200-300 DC, el desarrollo maduro de la Cultura San Pedro se situaría entre 200-300 DC hasta 900 DC. Incluso basándose en los aspectos alfareros, se han elaborado varias fases, dividiendo el desarrollo cultural de San Pedro en Fase I (Temprano o Formativo); en Fase II (Negro Pulido con Tiwanaku); Fase III (tipo Negro Casi Pulido) y en Fase IV (Rojo Violáceo, influencias altiplánicas tardías; tipo Negro Engobado Pulido en el interior de los pucos -tipo Dupont-). Esta Fase IV se subdividiría, a su vez, en una Fase Preinca y en otra inca (desde 1470 DC en adelante). Esta breve síntesis cronológica permite prever la importancia de la nueva fechación de Tiwanaku en San Pedro en la segunda mitad del primer milenio DC.

Además, la separación entre las Fases II y III se postulaba por la presencia o ausencia de la deformación craneana tabular oblicua (Munizaga 1969; Bittmann et al. 1978).

Nosotros continuaremos subdividiendo las fases del desarrollo de San Pedro de Atacama en tres. Sin embargo, subdividiremos la Fase II en dos subfases (a y b), privilegiando así la presencia de nuevos tipos alfareros (como el San Pedro Negro Casi Pulido). Por lo tanto, cuando mencionemos la Fase IIb nos estaremos refiriendo a la Fase III de otros arqueólogos. Nuestra Fase III equivale a la Fase IV de Bittmann y colaboradores (1978). c) En relación a la hipótesis de la presencia o ausencia de la deformación craneana tabular oblicua, concluimos en forma provisoria, apoyándonos en datos entregados por Le Paige (1963, 1964, 1965), que ella no coincide, en la mayoría de los casos, con la presencia de rasgos y artefactos Tiwanaku en San Pedro. Además, lo que es muy importante, la fechación relativamente tardía de Tiwanaku en San Pedro no permite relacionar en forma significativa la deformación tabular oblicua (especialmente representada en un cementerio, Quitor 2) con la cultura representada por la Fase II y que tiene artefactos y rasgos Tiwanaku.

\section{Desarrollo de nuestras hipótesis y su contrastación empírica}

Los cambios cronológicos postulados por nosotros se fundamentan en los siguientes datos: contextos provenientes especialmente de los cementerios Quitor, 1, 2, 3, 4, 5, 6, 7, 8 y 9, y algunas tumbas de Coyo Orlente, Tchecar, Tchilimoya y Toconao Oriente. Varias piezas calificadas por los investigadores Le Paige, Tarragó, Serracino y Núñez como "del más puro estilo Tiwanaku", "clásicas", o "Tiwanaku no transformado", se sitúan en un contexto cultural que contiene alfarería Negra Casi Pulida, alfarería tipo Dupont, tipo San Pedro Rojo Violáceo y formas de la alfarería San Pedro Negro Pulido, que pertenecen a desarrollos avanzados (tipo VII y decorado tipo B de Tarragó 1968, 1976, 1977) e, incluso, ceramios importados, altiplánicos y argentinos, que están fechados aproximadamente entre 800 y 1100 DC.

\section{Los huesos pirograbados}

Estos huesos pirograbados, que presentan figuras del "más puro estilo Tiwanaku" (figuras de hombres arrodillados con máscaras felínicas y alas, con cabezas cortadas, cetros y hachas en sus manos), han servido de fundamento, especialmente a Le Paige (1965) y Serracino (1980), para afirmar que "San Pedro de Atacama, como Chiripa y Wankarani, han dado el origen del mercado y el centro ceremonial de Tiwanaku" (Serracino 1980: 105). Incluso Le Paige ha escrito que la iconografía de los tubos pirograbados "es anterior a su ejecución en forma monumental pétrea", es decir, que los dibujos pirograbados en hueso, de Quitor 4, 5 y 6, son anteriores a la Puerta del Sol de Tiwanaku. Aparentemente las fechas de $C^{14}$ de Quitor 5 y 6 le daban la razón: 200 DC y 250 DC. 
Veamos con qué están asociadas estas piezas maravillosamente manufacturadas.

a) Un hueso pirograbado, encontrado en la tumba 2139-2140 en el cementerio de Quitor 5 (Le Paige 1965: Lám. 50) está asociado con tres tiestos alfareros del tipo Negro Casi Pulido tejidos de varios colores; una cuchara grande que tiene en el mango una figura de quirquincho; un pilón con felino que tiene entre sus patas una cabeza cortada; un hueso tallado en punta para tejer y una cabeza de auquénido.

b) Dos huesos pirograbados, encontrados en la tumba 2153 de Quitor 5 (Le Paige 1965: Láms. 51 y 52) asociados con piezas alfareras "rústica" y "negra tipo Tchecar", que corresponde al Negro Casi Pulido según Le Paige (1965: 70); con una cucharita de madera ordinaria, un disco metálico de $8 \mathrm{~cm}$ horadado y tejidos de varios colores.

c) Dos huesos pirograbados, de la tumba 1973-77 de Quitor 5 (Le Paige 1965: Láms. 53 y 54) asociados con una alfarería del tipo Negro Pulido, una tableta para rapé con bajorrelieve e incrustaciones, un tubo con cabeza de felino, tres cinceles de cobre, un arco quebrado, tres ganchos.

d) Un hueso pirograbado de la tumba 3592 de Quitor 6 (Le Paige 1965: Lám. 55) asociado con dos alfarerías Negras Casi Pulida, un vasito negro colorado, un huso con tortera de malaquita, un capacho destruido y dos caracoles de agua dulce.

e) Un hueso pirograbado de la tumba 1371-2 de Quitor 4 (Le Paige 1965: Lám. 56), asociado con alfarería común, calabazas, torteras de huso (Figura 2d).

f) Un hueso pirograbado con un diseño diferente a los anteriores (serpientes en forma de " $\mathrm{S}$ ", con dos cabezas en sus extremos), de la tumba 454 de Solor 3 (Le Paige 1965: Lám. 57), asociado con "tres vasitos colorados pintados de negro con asa de murciélago" y tres vasitos de alfarería Negra Pulida, un hacha de cobre, siete campanillas de cobre y varios caracoles de agua dulce.

Los contextos, aquí presentados por primera vez, permiten relacionar cuatro huesos pirograbados con alfarería de tipo Negro Casi Pulido, un hueso pirograbado con alfarería común y tres huesos asociados con alfarería Negra Pulida.
Si tenemos presente que la alfarería tipo Negro Casi Pulido constituye para todos los estudiosos de San Pedro una subfase tardía de la Fase II, y que para algunos correspondería a la Fase III, nos enfrentamos ante el hecho de que el 50\% de los huesos pirograbados del más puro estilo Tiwanaku llegaron (o se confeccionaron) en la segunda mitad del primer milenio de la era cristiana. ¿Qué ocurre con los otros huesos pirograbados? El hueso de Quitor 4 no tiene ningún artefacto asociado que lo sitúe en un tiempo más antiguo; los huesos de Quitor 5 asociados al tipo Negro Pulido (un tiesto) corresponden a una tumba colectiva $)^{2}$; y, por último, el hueso de Solor 3 , con motivos diferentes a los anteriores, parece ser el único que podemos situar en la Fase II.

Los kero de madera con decoración de rostro humano (vasos prosopomorfos)

a) Un kero prosopomorfo en tumba 2148 de Quitor 5 (Le Paige 1964: Lám. 127), sin contexto.

b) Un kero prosopomorfo en tumba 3874 , en Tchilimoya, asociado con un plato negro aislado (común) por fuera y Negro Pulido en el interior (tipo Dupont), restos de canastos, tres torteras de huso de madera y dos y media calabazas destruidas y sin dibujo.

c) Un kero prosopomorfo en tumba 3236 de Quitor 9, fechado con $\mathrm{C}^{14}$ en $1050 \mathrm{DC}$, asociado con cerámica Huruquilla (altiplánica) y un plato negro sobre rojo; dos tabletas de rapé; un vasito de piedra; un kero de plata (base); un punzón de cobre; una campana grande de madera (¿instrumento musical?) con una cruz grabada en su costado, etc.

Hay otro vaso prosopomorfo en este mismo cementerio (tumba 3241).

d) Resto de vaso prosopomorfo en Tchecar, sin contexto.

En Tchecar, según Le Paige (1954: 70), el piso inferior del túmulo principal contiene especialmente el tipo Negro Casi Pulido, "en sus tres formas robustas".

2 Metodológicamente investigamos tumbas individuales no removidas. Las fechadas por $\mathrm{C}^{14}$, en el caso de las tumbas colectivas, pierden valor, puesto que no se sabe qué parte del contexto fechan. Ver el caso de la tumba 2532-2546 de Quitor 6. 
También tenemos una variedad de tipos alfareros intrusivos; "los hay desde el tipo Tiahuanaco hasta el argentino-septentrional". "La nariz del centro de un kero clásico de Tiahuanaco, en greda rosada, fue encontrada desgraciadamente en terreno revuelto anteriormente."

Así los contextos de los vasos prosopomorfos pertenecen a un Período Tardío de la Fase IIb e incluso están asociados con cerámica del Período Tardío (Fase III) de San Pedro de Atacama. La fecha de $\mathrm{C}^{14}$ sitúa el tiempo entre 960 a 1140 DC (haciendo uso del \pm 90 ).

\section{Alfarería}

Son varias las piezas completas que relacionan al altiplano boliviano con San Pedro de Atacama; incluso algunas de ellas (kero) parecen haber sido hechas en San Pedro utilizando las técnicas regionales. Por ahora daremos solo algunos ejemplos.

a) Copa pintada de gris con dibujos geométricos de color negro y amarillo café, encontrada en la tumba 1330 de Quitor 2. Podría corresponder a un Tupuraya tricolor (Walter 1966), situado en un tiempo “Tiahuanaco Expansivo". Está asociada con cuatro alfarerías Negro Pulido (Figura 1a.4).

b) Pieza importada (vasito) de la tumba 1357 de Quitor 4, asociada con arcos quebrados, calabazas, cencerro de madera, caracol de agua dulce y concha plana marítima (Figura 1a.3).

Esta tumba está cerca de la que contiene el hueso pirograbado, asociada con alfarería común.

c) Un kero de color rojo de la tumba 4331 de Toconao Oriente, asociado con el tipo Negro Casi Pulido (una botella de cuello alto y de bordes evertidos y de paredes gruesas).

d) Un kero Negro Pulido de la tumba 1929 de Quitor 5, asociado con el tipo Negro Pulido (tipo VII de Tarragó 1966).

e) Tiesto keriforme de la tumba 3642 de Quitor 6, asociado a Negro Casi Pulido.
Kero de madera

a) Un kero de madera de la tumba 2241, de Quitor 5, asociado con cerámica tipo Isla (Tarragó 1977).

b) Un kero de madera de troncocónico, de base estrecha, con decoración de sobrerrelieve, serpentiforme, en la tumba 1117 de Quitor 1, asociado con cucharas de madera con espacio para suspensión en sus extremos y algunas horquillas de atalaje, de madera.

Este kero es relacionado con los kero de greda de Isla Parite, en el altiplano boliviano (Núñez 1963: 54).

Estos dos kero de madera poseen una situación relativamente tardía. Según Tarragó, la cerámica Isla debe situarse hacia 800 DC (a pesar de fechas de $C^{14}$ algo más tardías; Tarragó 1977). El kero con decoración serpentiforme "de influencia Tiwanaku", está situado en un conjunto de tumbas que, según Le Paige (1964: 75), deben situarse en el principio de la tercera fase del Agroalfarero atacameño.”

Complejo rapé

a) Tableta de la tumba 2189 de Quitor 5, con la escultura de un hombre enmascarado de felino, con cabeza cortada y hacha ("sacrificador") (Le Paige 1964: 2189-2192), asociada con alfarería tipo Negra Gruesa (Casi Pulida).

b) Tubos y tabletas de la tumba 3582 de Quitor 6, de estilo Tiwanaku, figura monolito con cabeza cortada y tableta con incrustaciones sobre fondo de oro, asociado con alfarería tipo Negra Casi Pulida.

c) Tableta de estilo Tiwanaku de la tumba 2748-49 de Quitor 6, asociada con alfarería tipo Negra Casi Pulida y Negra Incisa (Fase b o Tardía) (Orellana 1963; 17).

d) Conjunto de rapé con tableta con grabado de estilo Tiwanaku, asociado con alfarería tipo Negro Casi Pulido, en Coyo Oriente, tumba 5381.

Estos ejemplos-que pueden multiplicarse- muestran que figuras con decoraciones en estilo Tiwanaku, de gran calidad y belleza, están asociadas con elementos culturales de la Fase IIb de San Pedro de Atacama. 
Tejidos

a) Tejido que cubre la momia 4129 de Coyo Oriente, de estilo Tiwanaku, asociado a un tiesto alfarero (kero de Tiwanaku transformado o "expansivo", tipo Tchecar), asociado con una tableta, espátula, canastos, morteros (Figura 2a).

b) Fragmentos de tejido Tiwanaku de la tumba 4105 de Coyo Oriente, asociado con alfarería de tipo Negro Pulido (tipos VII y IX de Tarragó 1966), con tableta con cóndor, espátula de hueso y arco entero.

c) En Quitor 2 hay tejidos que pertenecen a las momias 61 y 65 (Figura 1b), asociados con alfarería tipo Negro Pulido, botelliforme (tipo "decorada" transición y tipo B, Tarragó 1966). Parece necesario recordar que Le Paige (1977: 123-24) nos informa que en Quitor 2 hay tumbas (5477-78 y 5469, 547072 con restos Tiwanaku asociados a alfarería tipo Negro Casi Pulido e incluso a alfarería tipo "Concho de Vino" (tumba 5477-78); en la tumba 5469 hay fragmentos de base de cerámica tipo Tchecar, asociados con Negro Casi Pulido (también en la tumba 5470-2).

Esto nos permite suponer que Quitor 2, importante por sus restos Tiwanaku, posee contextos pertenecientes a la Fase II, tanto a como b y que los tipos Negro Pulido pertenecen, en su mayoría, a la Fase IIb.

\section{Comentarios}

La primera consecuencia de nuestra hipótesis es que el número de piezas relacionadas con Tiwanaku y algunas otras culturas altiplánicas, situadas en la segunda mitad del primer milenio DC y comienzos del segundo milenio, es más abundante de lo que se estimaba hasta ahora (Serracino 1980). Podríamos comentar además que en la década de 1960 no hubo inconveniente en defender esta posición cronológica, pero que en la década siguiente se insistió en la presencia de rasgos Tiwanaku, situados tempranamente entre 200 y 500 DC (Ponce 1972; Bittmann et al. 1978). Es nuestra conclusión que algunas piezas del "más puro estilo Tiwanaku" se sitúan más tardíamente de lo supuesto hasta ahora por los especialistas, como es el caso de los "huesos pirograbados", algunas tabletas, tubos, tejidos y alfarería (Le Paige 1965).
Toda esta nueva situación cronológica tiene como segunda consecuencia que las fechas de $\mathrm{C}^{14}$, para las tumba 3397 de Quitor 5 y 2532-2546 de Quitor 6, no están fechando la llegada de Tiwanaku, ni la elaboración regional de piezas Tiwanaku, sino los comienzos de la Fase II de la Cultura San Pedro (200-250 DC).

Si esto es así, surge una tercera consecuencia: no encontramos evidencias claras de una nueva población altiplánica llegada hacia 200-400 DC (Bittmann et al. 1978: 41-42). El argumento de la deformación craneana tabular oblicua (Munizaga 1969) no ha sido apoyado por los datos que entrega el propio Le Paige (1964). Sólo parcialmente este tipo de deformación se relaciona en Quitor 2 con elementos y rasgos Tiwanaku; sin embargo, la tumba 1233 tiene este tipo de deformación sin asociación de ningún tipo con restos Tiwanaku. Algo parecido ocurre en Quitor 8. En Quitor 4, 5 y 6, en donde se encuentra el mayor número de evidencias Tiwanaku, es muy poca la presencia de este tipo de deformación; en Quitor 4 no hay, en Quitor 5 hay un 2.9\% y en Quitor 6 un $4.5 \%$. Esta misma hipótesis antropológico-física ha pretendido caracterizar la Fase II de la Cultura San Pedro, lo que no encuentra hasta ahora contrastación empírica. La diferencia entre San Pedro II a y b (o San Pedro II y III) no está dada por la presencia $o$ ausencia de deformaciones craneanas de los tipos tabular oblicuo y tabular erecto; ni tampoco la presencia o ausencia de estas deformaciones diferencias a poblaciones que confeccionaban el tipo alfarero Negro Pulido y el Casi Pulido.

Si estas inferencias nuestras se mantuviesen, tendríamos que pensar mejor el carácter de las relaciones altiplano-San Pedro de Atacama en donde, obviamente, el llamado estilo Tiwanaku es muy valioso, más que por su cantidad (aunque ésta no es desdeñable), por su calidad y significado ideológico. Sin aún manejar una teoría acabada, pensamos en un tipo de relaciones en donde lo comercial (el intercambio de materias primas y manufacturadas) y lo ideológico (el intercambio de ideas, de creencias) se efectuaban por la interacción de grupos de pastores y agricultores de la región de San Pedro de Atacama, con grupos que habitaban el Altiplano Sur boliviano y que, por supuesto, estaban influenciados por la civilización Tiwanaku.

Estos contactos y relaciones eran recíprocos y no cabe la menor duda que las influencias e intercambios se produjeron tanto desde San Pedro de Atacama al 
altiplano, como del altiplano al sur atacameño. Sin embargo, también es muy probable que la civilización Tiwanaku con su esfera de influencias (algunos estudiosos hablan de una "confederación altiplánica sur"; Browman 1980) haya modificado más a la sociedad de San Pedro de Atacama, especialmente en lo que se refiere al mundo de las creencias, que lo que ésta pudo hacer en sentido contrario.

Si descartamos el concepto de un Imperio Tiwanaku (Ponce 1972) y pensamos más en una sociedad bien organizada, altamente sofisticada y con un dominio político-económico e ideológico del sur del altiplano y de otras regiones, comprenderemos mejor el flujo de restos, rasgos e ideas desde el altiplano a San Pedro y sus alrededores, a través de caravanas, viajes de comercio, de peregrinajes a ciertos centros ceremoniales que iban y venían a lo largo de la segunda mitad del primer milenio DC.

Aunque hemos identificado algunas tumbas pertenecientes a personajes (jefes, chamanes), no creemos que ellas solas ejemplifiquen la llegada de productos y artefactos altiplánicos Tiwanaku, puesto que -como lo hemos mencionado- hay tumbas con contextos muy pobres que tienen sin embargo piezas importadas, o hechas de acuerdo al patron Tiwanaku en San Pedro mismo.

Así, las relaciones entre la sociedad de San Pedro de Atacama y las regiones de los lípez, chichas, uruquillas y otras situadas más al norte del altiplano, hasta alcanzar incluso a la propia ciudad de Tiwanaku (centro de todas las actividades comerciales y ceremoniales del altiplano), no son llevadas a cabo solamente por los jefes, para reafirmar su prestigio (Berenguer et al. 1980), sino por diferentes miembros de la sociedad interesados en intercambiar ciertos productos, tanto con fines económicos, como con objetivos ideológicos y de culto.
Para finalizar estas breves conclusiones provisorias, insistamos que ellas no están en contradicción con la presencia de algunos restos y rasgos Tiwanaku en San Pedro hacia 500 DC, tal como hace años atrás lo había señalado Uhle (Orellana 1982). Lo que hemos querido enfatizar es que el número mayor de restos, rasgos e ideas que llegan del altiplano se produce en un tiempo más tardío, cuando la cultura Tiwanaku había alcanzado su plena madurez y cuando la cultura San Pedro también estaba en condiciones de recibir mejor los elementos extranjeros.

Cuando la sociedad de Tiwanaku perdió parte del control del sur del altiplano (¿hacia 1100? DC), es probable que la cultura de San Pedro de Atacama haya jugado un papel importante en las relaciones comerciales e ideológicas, y en los intercambios de productos naturales y manufacturados con los pueblos de la región sur altiplánica de Bolivia y del norte argentino.

De acuerdo a los estudios de Fernández (1978) hay que insistir en las relaciones entre los habitantes de la Puna de Atacama y el sector limítrofe boliviano-argentino. Los yacimientos de Calahoyo y de Casira confirmarían los contactos directos entre San Pedro de Atacama, el extremo sur boliviano (Lípez y Chichas) y el extremo norte argentino. Estos intercambios, especialmente con la región sur altiplánica boliviana, están ejemplificados por diferentes tiestos alfareros encontrados en Tchecar Sur, en Quitor 2, 5, 6, 7 y 8, en Solor 3, etc., todos ellos pertenecientes al período de fines de Tiwanaku y postTiwanaku.

Agradecimientos A las autoridades de la Universidad del Norte, Antofagasta y, en especial, al Director del Museo Arqueológico de San Pedro de Atacama, Sr. Agustín Llagostera, las facilidades que nos dieron para investigar en el Museo fundado por el Padre Gustavo Le Paige. 
a

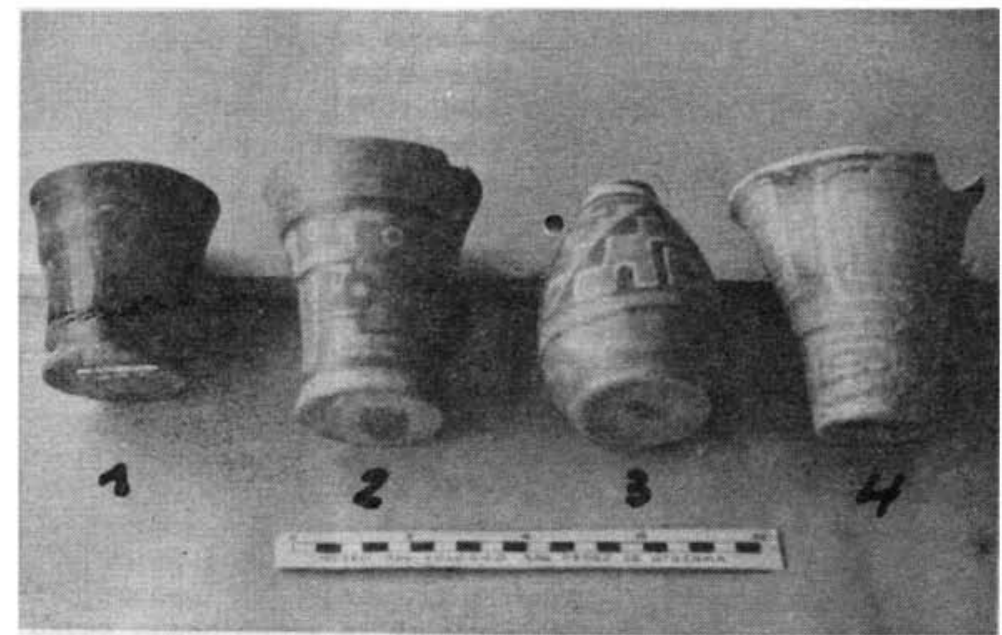

b

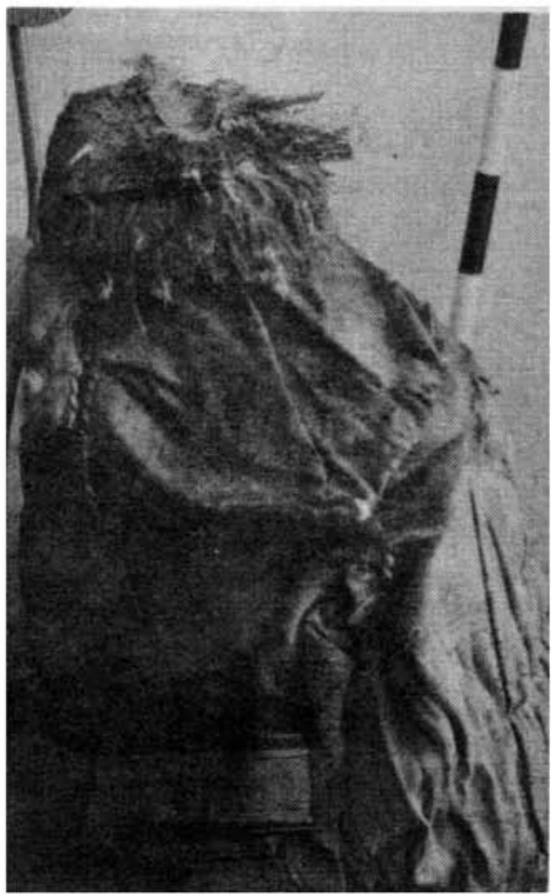

Figura 1. a1) Tipo kero Negro Pulido, Coyo Oriente, tumba 4060; a2) Tipo kero (Tiwanaku expansivo), Quitor 5, tumba 2246-7; a3) Tipo policromo (¿Tiwanaku Clásico?) Quitor 4, tumba 1357; a4) Tipo kero (postTiwanaku), Quitor 2, tumba 1330; b) Tumba 61 de Quitor 2, con tejidos Tiwanaku policromo, con decoración escalerada y de grecas. 


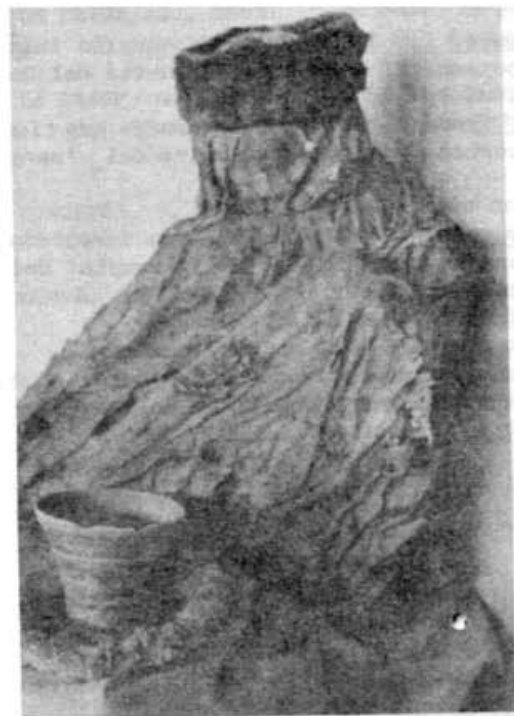

$\mathrm{a}$

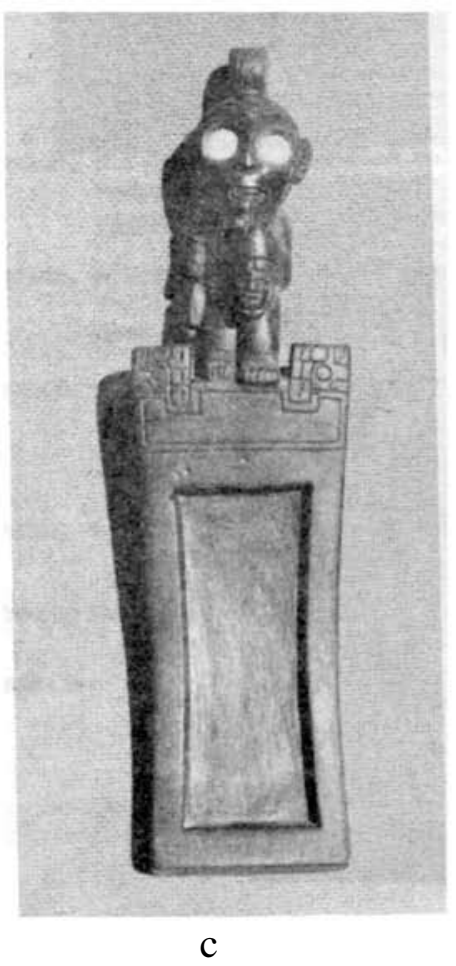

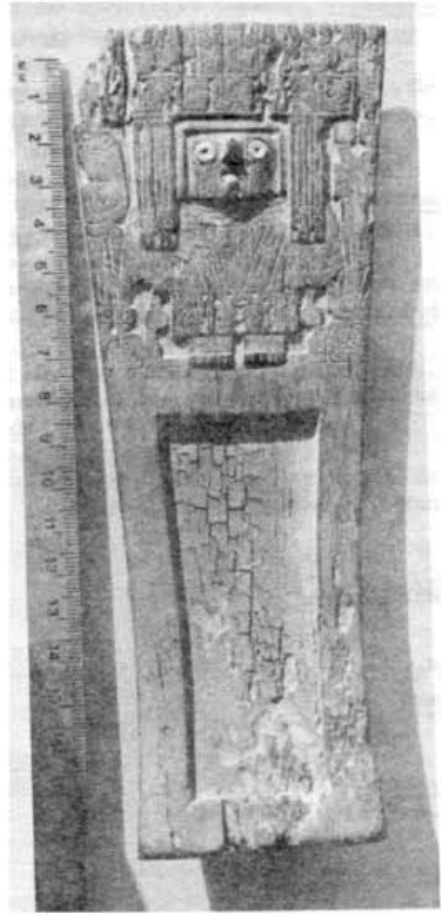

b

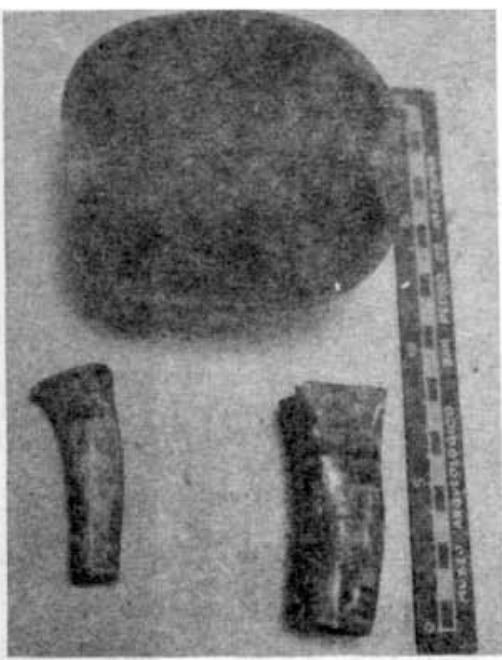

d

Figura 2. a) Momia de Coyo Oriente, tumba 4129, con tejido importado Tiwanaku; b) Tableta de Coyo Oriente, tumba 4093, con decoración inspirada en el personaje central de la Puerta del Sol de Tiwanaku; c) Tableta de Quitor 5, tumba 2196-7, con personaje que tiene una cabeza cortada y un hacha (motivo del "sacrificador"); d) Hueso pirograbado de Quitor 4, tumba 1371-2, con motivos inspirados en los personajes en cuclillas de la Puerta del Sol de Tiwanaku.

(*) Fotos autorizadas por el Instituto de Investigaciones Arqueológicas R. P. Gustavo Le Paige s. j., San Pedro de Atacama. 


\section{REFERENCIAS CITADAS}

BERENGUER, J., V. CASTRO y O. SILVA, 1980. Reflexiones acerca de la presencia de Tiwanaku en el norte de Chile. Estudios Arqueológicos 5.

BITTMANN, B., G. LE PAIGE y L. NUÑEZ, 1978. Cultura atacameña. Serie Patrimonio Cultural Chileno, Ministerio de Educación, Santiago.

BROWMAN, D., 1980. Tiwanaku expansion and altiplano economic patterns. Estudios Arqueológicos 5.

FERNANDEZ, J., 1978. Los chichas, los lípez y un posible enclave de la cultura de San Pedro de Atacama en la zona limítrofe argentino-boliviana. Estudios Atacameños 6.

LE PAIGE, G., 1963. La antigüedad de una tumba comprobada por $\mathrm{C}^{14}$ y el ambiente que la rodea. Revista Universitaria 26.

- 1964. El Precerámico en la cordillera atacameña y los cementerios del Período Agroalfarero de San Pedro de Atacama. Anales de la Universidad del Norte 3.

1965. San Pedro de Atacama y su zona (14 temas). Anales de la Universidad del Norte 4.

- 1977. Recientes descubrimientos arqueológicos en la zona de San Pedro de Atacama. Estudios Atacameños 5.

MUNIZAGA, J., 1969. Deformación craneana intencional en San Pedro de Atacama. Actas del V Congreso de Arqueología de Chile. La Serena.

MURRA, J., 1972. El control vertical de un máximo de pisos ecológicos en la economía de las sociedades andinas. Universidad Hermilio Valdizán, Huánuco.
NUÑEZ, L., 1963. Influencia de Tiahuanaco en la talla de madera del norte de Chile. Boletín de la Universidad de Chile.

— 1976. Registro regional de fechas radiocarbónicas del norte de Chile. Estudios Atacameños 4.

ORELlanA, M., 1963. La Cultura San Pedro. Centro de Estudios Antropológicos, Universidad de Chile, Publicación 17, Santiago.

1982. Investigaciones y teorías en la arqueología de Chile. Ediciones del Centro de Estudios Humanísticos, Universidad de Chile, Santiago.

PONCE, C., 1972. Tiwanaku: Espacio, tiempo y cultura. Academia Nacional de Ciencias de Bolivia, Publicación 30, La Paz.

SERRACINO, G., 1980. Tiwanaku desde San Pedro de Atacama Estudios Arqueológicos 5.

TARRAGO, M., 1968. Secuencias culturales de la etapa agroalfarera de San Pedro de Atacama (Chile). Actas y Memorias del XXXVII Congreso Internacional de Americanistas vol. 2. Buenos Aires.

1976. Alfarería típica de San Pedro de Atacama (norte de Chile). Estudios Atacameños 4.

1977. Relaciones prehispánicas entre San Pedro de Atacama (norte de Chile) y regiones aledañas: La Quebrada de Humahuaca. Estudios Atacameños 5.

WALTER, H., 1966. Beitrage zur Archaelogie Boliviens. Berlín. 